\title{
LE CLOÎTRE ET LA LETTRE : LECTURE DE LA CORRESPONDANCE D'HÉLOÏSE ET D'ABÉLARD
}

Karen Aldrich, Université de Victoria.

\section{Résumé analytique}

La réalité de l'enfermement peut prendre plusieurs formes: physique, spirituelle, matérielle; pour Héloïse et Abélard, célèbre couple du XII ${ }^{\mathrm{e}}$ siècle, le confinement est à la naissance de l'écriture : leurs lettres, échangées après leur séparation et lorsqu'ils sont, chacun, à la tête d'une abbaye, ne visent pas à dépasser l'absence ni à la nier, pas plus qu'elles ne veulent briser l'enfermement; au contraire, elles s'inscrivent dans l'espace clos de l'abbaye et en définissent les règles de la vie hors du monde.

La lettre, moyen de communication à la fois personnel et public, est un espace de liberté littéraire : c'était parfois la seule façon qu'avait la femme de sortir dans le monde, parfois la seule possibilité qu'avait un homme d'entrer en communication avec une femme érudite, qui n'était pas son épouse. Ainsi, la lettre doit sortir de l'enfermement ; ici, elle se métamorphose en une célébration du même enfermement et devient le d'une fructueuse libération intérieure.

\section{$* * *$}

Toute écriture est une forme de confinement, une manière de détenir les idées qui viennent pour leur part, d'un isolement intérieur de celui ou celle qui les écrit, ou les laisse écrire. Mots encadrés sur la page, serrés par les marges blanches, l'écrit devient prisonnier jusqu'au moment où le lecteur le lit et le fait entrer dans sa conscience. Le mot trouve sa liberté au moment où celui ou celle qui l'a lu le métamorphose en une notion vivante et le remet en écriture dans la reconfiguration, la reconstruction d'une réponse. Voici, alors, que le cycle d'enfermement et de libération commence de nouveau et continue potentiellement à perpétuité, pour arriver au sommet de sa fécondité.

De fait, si l'on conçoit le corps comme contenant, chacun vit dans un enclos. Cette métaphore structurelle a été élaborée surtout au Moyen Age dans le domaine de la dévotion religieuse. Le lien pourrait être simplement décrit par les termes suivants: on sort de la réalité intérieure, on la rend visible en la transmettant en situation matérielle et externe: on enferme le corps, comme l'âme et l'esprit sont déjà enfermés à l'intérieur du corps - c'est-à-dire, le bâtiment du cloître ou monastère qui enferme, devient la réalité visible de ce que l'esprit a déjà subi ${ }^{1 .}$ Ainsi, la façon monastique de vivre peut être comprise comme une existence pensive, tournée vers l'intérieur, dont la dimension invisible se reflète dans les implications métaphoriques du lien direct avec le corps, ou la vie enfermée. Or l'essence de l'existence, et sa dualité entre intérieur et extérieur sont mises à l'essai, dépassées et épurées².

Alors que le mot est prisonnier de la page, de l'oeuvre, le corps emprisonne l'âme, l'intellect et vice- versa. Lettre et corps accèdent ainsi à un statut comparable; la lettre comme le corps humain

$\ldots$ is constituted in complex material and analogical relations, interacting with psychic forms, geographical space, and the social formation. The body [and by extension the letter] cannot, then, be thought of separately from social topography or from an imaginary corporeal morphology that is complexly regulated, negotiated, and contested through the body's interrelations with place and discourse ${ }^{3}$.

Au Moyen Age, c'est avec acuité intellectuelle qu'est pensé l'emmurement scripturel et corporel, y compris dans ses implications psychiques, sociales et géographiques. A l'époque, la lettre est toujours temporellement proche de son origine comme message oral (mot libre), devenu écrit (mot enfermé). Le voyage, la distance faisaient de la parole du messager humain une impossibilité, et le message transmis est devenu une écriture entre deux personnes qui était à 
l'époque

...half of a conversation or dialogue between the sender and the addressee, and it involved a quasi presence and quasi-speech between the two ${ }^{4}$.

Or, la lettre au moyen âge est un moyen de communication à la fois personnel et public, elle devient un espace ou de liberté littéraire, ou de littérature libératrice: c'était parfois la seule façon qu'avait la femme de sortir dans le monde, parfois la seule possibilité qu'avait un homme d'entrer en communication avec une femme érudite, qui n'était pas son épouse. Ainsi, la lettre devient la présence corporelle de l'autre; elle devient la personne-même qui peut maintenant sortir de l'enfermement tout en célébrant ce même enfermement. Dans la correspondance d'Abélard et d'Héloïse, par exemple, la lettre devient effectivement le moyen de s'approcher de loin, de donner un confort mutuel, et l'agencement d'une fructueuse libération intérieure 5 .

Huit lettres consécutives, (peut-être ou peut-être pas écrites par Pierre Abélard et Héloïse entre 1128 et $1142{ }^{6}$ ), constituent la collection de cette correspondance en un ensemble, une unité de textes qui visent et décrivent un grand mouvement, notamment la progression intérieure et extérieure des séquestrations respectives vers une émancipation complète des deux écrivains. La première lettre, l'Historia calamitatum de Pierre Abélard, est écrite comme message théologique et autobiographique venant de l'autorité de sa propre expérience. D'après Constant Mews, on y trouve un chef-d'oeuvre de l'art narratif:

Abelard's account is itself a masterpiece of narrative art in which specific experiences, presented with great detail, are used to illuminate an original theological argument intended to be universal in application?

Bien que l'application doive en être universelle, l'attention est premièrement portée sur la situation dans laquelle il se trouvait au passé du récit, se trouve au présent, les circonstances qui l'emprisonnaient et l'enfermaient intérieurement et, par conséquent, dans son action extérieure. Son existence, encadrée dès sa naissance par une "certaine légèreté d'esprit" 8 inhérente, est pénétrée par la passion pour l'étude. Personnifiée comme séductrice, il devient sa victime à cause du "charme qu'elle exerça sur [s]on esprit"'. Lors de cette séduction, il brille en matières académiques, on le trouve intelligent, il s'enfle d'orgueil et devient par suite enchaîné. Il est "dévoré par la fièvre de l'orgueil et de la luxure" 10 , car

...la prospérité enfle toujours les sots; la sécurité de ce monde énerve la vigueur de l'âme et en brise facilement les ressorts par les attraits de la chair ...11

Abélard décrit son penchant narcissique, ses motivations égoïstes par rapport aux débuts de sa relation avec la jeune Héloïse, en une description entrelacée de projections de ses succès philosophiques comme maître de sa propre école de pensée. Ce succès passé est vu, par un Abélard plus mûr, non pas tant comme un sommet que comme le début de la chute:

Tel fut le commencement de la série de mes malheurs, qui durent encore: ma renommée grandissant chaque jour davantage, l'envie des autres s'alluma contre moi ${ }^{12}$. 
Les mots "commencement" et "durent encore" soulignent le fait qu'il ne peut en sortir, qu'il vit dans la continuation de ce qui a commencé jadis.

L'épisode transformateur a pour objet narratif la tragédie de sa castration par la famille d'Héloïse, suivie par son entrée au cloître de St. Denis. D'un jour à l'autre, il subit deux genres d'enfermements physiques qui semblent être les plus difficiles des restrictions: la perte de la liberté sexuelle dans la vie intime et la perte du mouvement libre dans la vie sociale. Cependant, c'est en vérité son appui constant sur ses déboires passés qui représentent le plus grand emprisonnement. Il se laisse subjuguer par les regrets; il devient, d'après sa narration, de plus en plus asservi au temps: il est l'esclave d'un passé qui glisse sur la situation de son présent et le paralyse. Ne pouvant oublier son effondrement académique, les castrations publiques soit corporelle, soit intellectuelle (la destruction de son livre pour les motivations politiques contre sa personne), l'échec de sa carrière dans un monastère peuplé de moines décrits comme débauchés, il livre au présent le grand désespoir qu'il ressent au moment de l'écriture:

Errant, fugitif, il semble que je traîne la malédiction de Caïn . Je le répète, "au dehors les combats, au dedans les craintes", me tiennent incessamment en proie; bien plus, audehors comme au-dedans ce sont autant de combats que de craintes ${ }^{13}$.

Puisqu'Abélard est pris au piège dans l'enclos de sa mémoire, de son corps, de sa situation anachorétique, sa lettre à un ami souffrant lui permet un affranchissement qu'il ne peut effectuer seul. Ses limitations corporelles du moment reflètent l'état de son esprit qui précède la rencontre avec Héloïse; elles deviennent le moteur de sa rédemption, son défaut physique devenant la pierre de gué menant à sa délivrance interne.

Héloïse, abbesse du couvent du Paraclet, par contre, est déjà libre. Cloîtrée depuis longtemps elle aussi, bien que ce fût par la volonté d'Abélard après leur mariage, ce n'est point qu'elle ne lutte pas contre la tension entre une vocifération corporelle et spirituelle - elle l'a acceptée et a transformé son énonciation en écriture: elle écrit sur son corps, écrit sur ses pensées, sur son esprit, bref, elle s'écrit, et en s'écrivant elle attire l'Abélard engourdi vers sa propre liberté. Née femme, Héloïse n'avait jamais eu le privilège de se donner à l'illusion d'une liberté, comme avait pu le faire Abélard en tant qu'homme. Il n'a pas pu y avoir, donc, le même palier de déplacement, d'effondrement. Femme érudite et intelligente à l'époque où peu d'hommes et encore moins de femmes avaient accès à une éducation, elle a dû tôt accepter qu'elle était seulement libre dans la situation cloîtrée de la vie monastique. La "liberté", hors les murs du couvent, ou le mariage auraient été la vraie prison, et toute son éducation perdue. Châtrée figurativement depuis et à cause de sa naissance féminine, elle connaît seulement la réalité d'être eunuque soit parmi les femmes à cause de son niveau d'éducation, soit parmi les hommes à cause de son sexe.

Capable et intelligente, Héloïse

found herself circumscribed in a textual culture in which literacy was obtained through a male rite of passage in the grammatical schools. Participation in textual culture and the performance of literate practices were encoded as masculine. The literate person, whose distinctive subjectivity was constituted by the participating in this culture was known only by the masculine name in Latin, the litteratus ${ }^{14}$. 
Donc, Héloïse a toujours vécu à l'étroit dans les limites d'une culture qui ne voulait lui donner l'égalité dont elle jouissait dans la légitimité effective de son intelligence. Élevée avec une formation masculine et dans une situation sociale qui ne valorisait point son sexe, sauf dans la fiction de l'amour courtois à jamais inaccessible, elle se trouve, à l'époque de la correspondance, dans une meilleure situation que celle d'Abélard, car elle a déjà dû apprendre à vivre avec la contrainte. De plus, elle a pu, grâce à son entrée au cloître, négocier l'acceptation de son travail sans les pressions carriéristes subies par les clercs de son époque. Ses lettres reflètent cette liberté, car

( $n$ )ot only do Heloise's letters reveal a highly skilled writer, but one who negotiated a new space within literate culture - that of the woman as litteratus and friend (amicus). Heloise in her letters negotiated and redefined her identity and subjectivity to write like a litteratus and as a woman ${ }^{15}$.

Ceci n'a pu être possible qu'à cause de son enfermement dans le couvent d'Argenteuil et finalement au Paraclet. Là, elle était libre de suivre ses études, d'augmenter les intérêts du couvent, d'y développer une réputation d'érudition indépendante ${ }^{16}$. Elle s'était éloignée, pour la plus grande partie, des resserrements et empêchements séculaires du monde extérieur au cloître. Conséquemment, il n'y avait plus se retenir, et son écriture renvoie à une femme qui accepte le passé, qui est solidement soutenue dans le présent, et qui porte un regard confiant vers le futur.

Dans la suite de la correspondance, Héloïse commence avec sa perspective personnelle et son propre commentaire sur les événements décrits par Abélard dans son Historia calamitatum. Héloïse, discernant astucieusement la profondeur de l'emprisonnement d'esprit d'Abélard et son affaissement, continue en le sommant de s'engager: elle le fait sortir de son apitoiement sur lui-même, et l'anime dans la réalisation de ses responsabilités envers "sa servante..., sa fille..., son épouse..., sa soeur..." 17 et l'abbaye qu'il a fondée. Elle l'éveille de son sommeil avec son honnêteté aiguë par rapport à son corps, son esprit et son âme, elle l'attire enfin vers le processus libérateur où il sera forcé de penser hors de son enclos d'une manière également spirituelle:

....mais quel que soit l'objet de tes lettres, elles ne laisseront pas de nous faire un grand bien, par cela seul qu'elles seront une preuve que tu ne nous oublies pas ${ }^{18}$.

La tactique d'Héloïse est simple: utiliser la situation traditionnelle masculine envers la femme pour que la femme finisse par sauver l'homme.

For the $12^{\text {th }}$ century litterati, gender classes seemed to be natural categories mirrored in language, and maleness and femaleness would have been understood as both natural and social identities... Heloise's strategy of negotiation and new self-definition utilized the gender categories of the $12^{\text {th }}$ century $\ldots{ }^{19}$.

En s'inscrivant dans le récit d'Abélard, c'est-à-dire en ajoutant sa propre perspective (qui est nécessairement étroitement liée à celle de son époux), elle suscite une réaction de sa part et le mène à une réponse. La description par Héloïse de son supplice cause la réaction voulue: Abélard se montre à la hauteur de la situation, et relève le défi d'encourager et de guider, et à tour de rôle de se laisser encourager et guider. Ainsi, il parvient à sortir des murailles intérieures qu'il s'est construites. 
Les réponses d'Abélard rendent visible la transformation qui a eu lieu dans la disposition de son esprit et qui se lit premièrement dans la valorisation d'Héloïse; le rapprochement vers elle et vers son travail comme abbesse au Paraclet; et la tendresse avec la supplication pour les prières (troisième lettre ${ }^{20}$ ). La demande de la prière vient d'une intimité fondamentale, elle devient la première étape vers l'acceptation que sa femme puisse être un "amicus", une collègue égale devant Dieu. Au lieu de se plaindre, il peut enfin se détacher de sa situation et voir le positif dans le malheur d'autrefois. Il rejette alors son impossible joug et accepte son passé comme richesse ${ }^{21}$. La souffrance devient salut; à la fin de la cinquième lettre ${ }^{22}$, il arrive finalement à ne plus demander, mais à donner, sous la forme d'une prière écrite pour Héloïse.

Y a-t-il une séparation à faire entre les "lettres d'amour" et les "lettres théologiques, religieuses"? Il y a, depuis des siècles, des critiques qui visent cette division, et depuis le 17ème siècle on a souvent séparé les deux parties qu'on pensait être déséquilibrées. On pourrait par contre les considérer comme un échange épistolaire ayant une unité thématique du début à la fin, comme un courant continuel qui commence à un point de départ et finit autre part, chenal libéré par Héloïse lorsqu'elle a répondu à l'Historia calamitatum ${ }^{23}$. Les premières lettres commencent avec le soin et l'exhortation intime au niveau émotif, les dernières finissant en démontrant un soin pragmatique et intellectuel qui dépassent l'attention des premières en ce qu'elles puisent d'une intimité qui sort d'une genèse plus élevée: celle qui a sa source en Dieu. L'évolution de la thématique des lettres n'est point une division, mais un apogée, un comble qui se développe naturellement de l'égalité qui se manifeste dans la relation entre Abélard et Héloïse. Cette égalité fait des dernières lettres (les sixième à huitième lettres) le résumé du complet de leur liaison conjugale et amicale. L'échange acquiert un équilibre duquel les deux personae peuvent s'enrichir: chacun termine par recevoir ce dont il/elle a besoin de l'autre - la reconnaissance de son auctoritas, de son droit d'autorité ${ }^{24}$.

\footnotetext{
...Abelard's later letters mark the culmination - emotional as well as intellectual and spiritual - of his relationship with Heloise: their shared involvement in the project of monastic foundation and reform ${ }^{25}$.
}

Cette continuation est effectivement une synthèse des tractations d'auparavant - les deux parties séparées se sont rapprochées, elles se sont réunies dans leur parité, et ni la distance géographique, ni l'enclaustration ne peuvent plus les diviser. On arrive à la fin de la série des lettres, qui culminent dans la mise en place d'une série de règles pour l'abbaye du Paraclet. C'est une oeuvre produite en collaboration, et qui devient leur jonction: Héloïse fait la demande en prescrivant ce qu'elle pense nécessaire, elle donne ses raisons en citant les écrits faisant autorité; Abélard réagit en respectant les arguments d'Héloïse, en les mettant sur papier et en leur conférant autorité. Conçu en particulier pour régler les problèmes de la réalité de l'enferment monastique féminin, fondé dans l'amour profond qu'a Héloïse pour les femmes qui dépendent d'elle, le travail sur les règles encourage Abélard à s'inscrire dans l'ordonnance d'une organisation déjà réussie. Abélard prend le flambeau et construit les Règles du Paraclet. Or, ensemble, Abélard et Héloïse créent une tâche accomplie avec, à travers, et par l'amour, une tâche qui surpasse toutes les difficultés individuelles et jointes, et qui devient un travail qui échappe à l'enclos des lettres et du temps pour rendre témoignage de ce qu'ils ont pu accomplir conjointement dans le service de leur Dieu.

http://www.brocku.ca/cfra/voixplurielles06-01/index.html 
Leur succès est démontré par le fait que les lettres deviennent par la suite des textes d'espoir, textes du renforcement entre deux égaux qui partagent maintenant non seulement un passé commun, mais en plus un présent dans l'écriture, qui vise en fin de compte un futur inépuisable. Les lettres transforment le présent enfermé en activité fructueuse dans la reconstruction d'une fois leur relation, de l'autre la structure du Paraclet (ou la besogne du Ciel). Le mariage et le monastère, également accablés par une discipline trop rigoureuse, par des prescriptions trop forcées, ont besoin d'un renouvellement. La prise de conscience de cette nécessité est la continuation de ce qu'Héloïse a commencé à faire dans sa première réplique à Abélard.

Le but des dernières lettres devient alors de restituer la relation monastique aussi bien que la relation intime, bien qu'en structures différentes, afin de libérer les deux personnes, non des liens d'amitié, mais des liens restrictifs de l'amitié née d'une passion charnelle, et qui est restée dans la mémoire comme liaison douloureuse. C'est l'évolution de l'amour ravalé, figé dans le souvenir subjectif de chaque individu impliqué, qui devient finalement un amour libéré et basé sur la profondeur d'esprit qui échappe à la souffrance, au manque et atteint la méditation et l'accomplissement d'une égalité d'esprit ainsi que le respect mutuel qui s'ensuit. Abélard est celui qui progresse de la manière la plus dramatique. Héloïse avouait déjà au début de la correspondance que son amour était beaucoup plus généreux que celui de son mari. Bien qu'elle semble se conformer au moeurs du temps en s'adressant à lui comme "maître", ceci semble être simple rituel pour qu'Abélard se rende compte du déséquilibre qui existe, pour qu'il s'élève au-dessus de soi-même et qu'il puisse dépasser les moeurs et la tradition dans son "zealous pugnacious piece, exuding energy and a sense of discovery" 26 sur l'autorité et la dignité des religieuses, c'est-à-dire, des femmes ${ }^{27}$ (huitième lettre).

Abelard, (...), even though far from feminist in his underlying assumptions about women's "weakness" as shown earlier, perceived a proto-feminist way to construct a fresh reading of Scripture by identifying and emphasizing - as if from a female subject position - priority of female rather than male achievements ${ }^{28}$.

Cette lettre exprime non seulement une érudition profonde en matière biblique, mais de plus l'égard, l'estime immense qu'Abélard porte à Héloïse comme femme et personne d'autorité. Il nivelle un plan qu'Héloïse avait déjà aplani depuis des années avec son travail à l'abbaye. Voici alors le rétablissement d'un mariage à un niveau beaucoup plus élevé. Abélard et Héloïse, époux et épouse; abbé et abbesse; citoyens terrestres avec tous les problèmes qui s'attachent à la condition humaine; deux individus comparables dans tout sauf leur sexes, sont d'ores et déjà émancipés dans leur relation l'un à l'autre et dans leurs positions respectives pour pouvoir s'approcher librement et sans contraintes à travers leur foi, leur humanité et leur intelligence.

À la fin de la correspondance, il n'y a en fait plus rien à écrire. Ils ont, en écrivant les huit lettres, traversé le terrain qui leur permet d'être liés pour toute l'éternité: libérés des préjugés sociaux, comme d'une temporalité linéaire enchaînant passé, présent et futur, Abélard et Héloïse se trouvent pour toujours dans le cycle de la continuation potentiellement perpétuelle: le cycle de l'enfermement libérateur et de l'écriture. Ils sont en effet passés de la vie restreinte par la corporalité à la dimension 
du temps de Dieu, l'éternité.

La Correspondance rejoint, en effet, la méditation de saint Bernard sur l'incarnation, l'affirmation par la mystique cistercienne que l'homme est fait d'abord de chair, qu'il faut donc partir de la chair, saisir en ses sources corporelles la pulsion amoureuse, l'endiguer, en guider patiemment le cours afin qu'elle devienne le moteur d'une ascension spirituelle ${ }^{29}$.

Le corps lourd est devenu l'idée légère qui se libère de nouveau chaque fois qu'elle est lue.

\section{Ouvrages cités et consultés}

Abélard et Héloïse. Correspondance. Édouard Bouyé, ed. France: Gallimard, 1938, 2000. (AH)

Beckwith, Sarah. "Passionate Regulation, Enclosure, Ascesis, and the Feminist Imaginary". South Atlantic Quarterly 93.4 (1994): 803-804.

Blamires, Alcuin. The Case for Women in Medieval Culture. Oxford: Clarendon, 1997.

Baswell, Christopher. "Heloise". Dinshaw et Wallace 161-171.

Cannon, Christopher. "Enclosure". Dinshaw et Wallace 109-123.

Charrier, Charlotte. Héloïse dans l'histoire et dans la légende. Paris: Librairie Ancienne Honoré Champion, 1933.

Constable, Giles. Letters and Letter Collections. Turnhout: Éditions Brepols, 1976.

Copeland, Rita. Criticism and Dissent in the Middle Ages. Cambridge: Cambridge UP, 1996.

Dinshaw, C., D. Wallace, eds. The Cambridge Companion to Medieval Women's Writing.

Cambridge: Cambridge UP, 2003

Dronke, Peter. Women Writers of the Middle Ages. Cambridge: Cambridge UP, 1984. Abelard and Heloise in Medieval Testimonies. Glasgow: Glasgow UP: 1976.

Duby, Georges. Dames du XIIè siècle. Mayenne: Gallimard, 1995.

East, W.G. "This Body of Death: Abelard, Heloise and the Religious Life". Biller, Peter, A.J. Minnis, eds. Medieval Theology and the Natural Body. Rochester: York Medieval press, 1997.

Ferrante, Joan. To the Glory of her Sex. Indianapolis: Indiana UP, 1997.

Freeman, Elizabeth. "The Public and Private Functions of Heloise's Letters". Journal of Medieval History 23.1 (1997): 15-28.

Griffiths, Fiona. "'Men's duty to provide for women's needs': Abelard, Heloise, and their negotiation of the cura monialium". Journal of Medieval History 30 (2004): 1-24.

Irvine, Martin. "Heloise and the Gendering of the Literate Subject". Copeland 87-114. 
Rougemont, Denis de. L'Amour et l'occident. Paris: Plon, 1939.

Lundt, Bea. Ed. Auf der Suche nach der Frau im Mittelalter. München: Wilhelm Fink, 1991.

McNamer, Elizabeth. The Education of Heloise. Lewiston: Edwin Mellen Press, 1991.

Mews, Constant. Abelard and Heloise. Oxford: Oxford UP, 2005.

Moos, Peter von. "Die Epistolae duorum amantium und die 'saekulare Religion der Liebe' ". Studi

Medievali 44 (2003): 1-115.

Nichols, Stephen. "Medieval Women Writers: Aisthesis and the Powers of Marginality". Yale

French Studies 75 (1988): 77-94.

Summit, Jennifer. "Women and Authorship". Dinshaw et Wallace 91-108.

Cannon, Christopher. "Enclosure". Dinshaw et Wallace, p. 109.

Cannon, Op.Cit., p. 110.

Beckwith, Sarah. "Passionate Regulation, Enclosure, Ascesis, and the Feminist Imaginary".South Atlantic Quarterly 93.4 (1994), p. 804.

Constable, Giles. Letters and Letter Collections. Turnhout: Éditions Brepols, 1976, pp. 12-13.

Constable, Op.Cit., p. 33.

McNamer, Elizabeth. The Education of Heloise. Lewiston: Edwin Mellen Press, 1991, p. 6.

Mews, Constant. Abelard and Heloise. Oxford: Oxford UP, 2005, p. 150.

Abélard et Héloïse. Correspondance. Édouard Bouyé, ed. France: Gallimard, 1938, 2000, p. 57.

Abélard et Héloïse, Op.Cit., p. 57.

10 Abélard et Héloïse, Op.Cit., p. 60.

11 Abélard et Héloïse, Op.Cit., p. 60.

12 Abélard et Héloïse, O.Cit., p. 58.

13 Abélard et Héloïse, Op.Cit., p. 106.

14 Irvine, Martin. "Heloise and the Gendering of the Literate Subject". Copeland, p. 87.

15 Irvine, OP.Cit., p. 87.

16 McNamer, Op.Cit., p. 2.

17 Abélard et Héloïse, Op.Cit., p. 111.

18 Abélard et Héloïse, Op.Cit., p. 113.

19 Irvine, Op.Cit., p. 107.

20 Abélard et Héloïse, Op.Cit., pp. 122-130.

21 Abélard et Héloïse, Op.Cit., pp. 157-158.

22 Abélard et Héloïse, Op.Cit., pp. 143-167.

23 Blamires, Alcuin. The Case for Women in Medieval Culture. Oxford: Clarendon, 1997, p. 201.

24 Blamires, Op.Cit., pp. 201-202.

25 Griffiths, Fiona. "' 'Men's duty to provide for womenès needs': Abelard, Heloise, and their negotiation of the cura monialium". Journal of Medieval History 30 (2004), pp. 2-3.

26 Blamires, Op.Cit., p. 202.

27 Abélard et Héloïse, Op.Cit., pp. 248-352.

28 Blamires, Op.Cit., p. 204.

29 Duby, Georges. Dames du XIIè siècle. Mayenne, Gallimard, 1995, p. 103. 